\title{
Congenital Diaphragmatic Hernia Presenting with Tension Pneumothorax in a 3-Year-Old Boy
}

\author{
Maren Friederike Balks ${ }^{1} \quad$ Jan-Hendrik Gosemann ${ }^{1} \quad$ Ina Sorge ${ }^{2}$ Martin Lacher ${ }^{1} \quad$ Franz Wolfgang Hirsch ${ }^{2}$ \\ ${ }^{1}$ Departement of Pediatric Surgery, Universitatsklinikum Leipzig, \\ Leipzig, Sachsen, Germany \\ ${ }^{2}$ Departement of Pediatric Radiology, Universitatsklinikum Leipzig, \\ Leipzig, Sachsen, Germany \\ Address for correspondence Maren Friederike Balks, MD, \\ Department of Pediatric Surgery, University of Leipzig, Liebigstraße \\ 20 A, Leipzig 04103, Germany \\ (e-mail: maren.balks@medizin.uni-leipzig.de).
}

Eur J Pediatr Surg Rep 2018;6:e63-e65.

\begin{abstract}
Keywords

- tension

- pneumothorax

- congenital

- diaphragmatic

- hernia

We report the case of a 3-year-old boy who presented with an upper respiratory tract infection and severe dyspnea. A chest $\mathrm{X}$-ray revealed a left-sided tension pneumothorax with mediastinal shift and suspected enterothorax. After thoracic computed tomography (CT) scan, a chest tube was inserted, which drained fluid which had the same consistency and color as the one derived from the nasogastric (NG) tube. The boy underwent diagnostic laparoscopy for suspected bowel perforation, which confirmed a left-sided Bochdalek hernia with herniation of the viscera into the chest. After repositioning of the herniated organs into the abdomen, a gastric perforation was identified and repaired. This case demonstrates that the cause of a tension pneumothorax in an infant may be a rare combination of congenital diaphragmatic hernia $(\mathrm{CDH})$ and perforation of a visceral hollow organ.
\end{abstract}

\section{New Insights and the Importance for a Pediatric Surgeon}

$\mathrm{CDH}$ may be asymptomatic at birth and may present at a later age. The herniation of the viscera into the chest leads to respiratory symptoms. In case of both, enterothorax and tension pneumothorax, a gastric or bowel perforation in combination with $\mathrm{CDH}$ must be taken into consideration.

\section{Introduction}

Congenital diaphragmatic hernia $(\mathrm{CDH})$ is a birth defect of the diaphragm caused by an insufficient closure of the pleuroperitoneal canal during fetal development. ${ }^{1}$ About $80 \%$ of these malformations are located on the left posterolateral side and are therefore called Bochdalek hernias. ${ }^{2}$ Most patients with $\mathrm{CDH}$ are diagnosed prenatally or immediately after birth. ${ }^{3}$

However, children with small defects may be asymptomatic postnatally and present later in life, when an increased intraabdominal pressure facilitates herniation of organs into the chest. $^{4-6}$ Some cases of $\mathrm{CDH}$ develop gastrointestinal symp- toms due to gastric ischemia or injury or volvulus. ${ }^{3,7}$ We report on a child with previously unknown Bochdalek hernia and lifethreatening presentation due to tension pneumothorax.

\section{Case Report}

A 3-year-old boy presented to an outside hospital with a 5day history of progressing respiratory distress and retching. The initial chest X-ray showed a left-sided tension pneumothorax with mediastinal shift and the suspicion of bowel loops in the left lower hemithorax (-Fig. 1). Therefore, the patient was transferred to our institution.
License terms

Stuttgart · New York received
June 29,2018

accepted

July 3, 2018

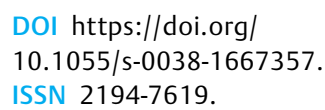




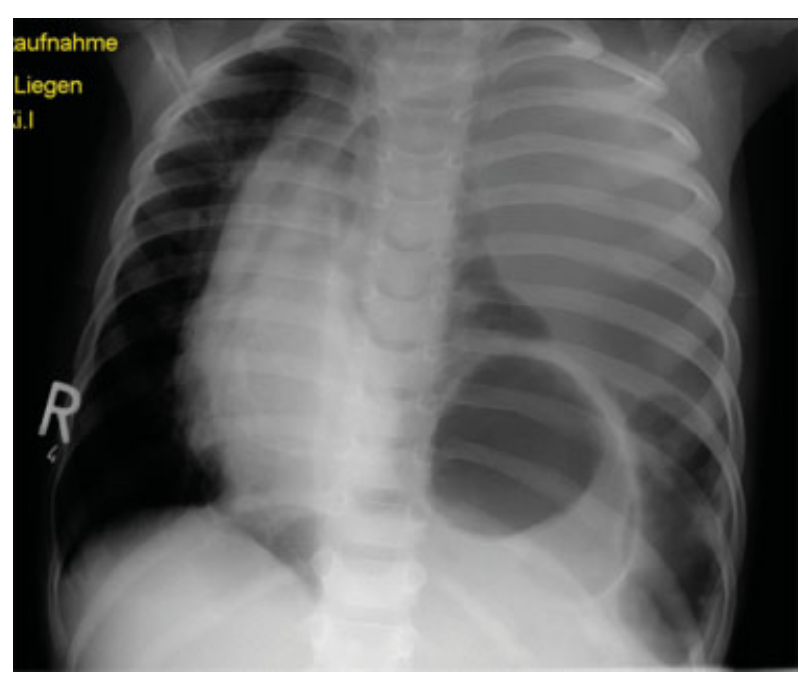

Fig. 1 Chest X-ray at presentation (This image is provided courtesy of Pediatric Clinic Fachkrankenhaus Hubertusburg, Wermsdorf).

On admission, he showed severe dyspnea, a temperature of $39.5^{\circ} \mathrm{C}$ and tachycardia of $200 / \mathrm{min}$. After immediate endotracheal intubation, a thoracic computed tomography (CT) scan was performed, which confirmed a left-sided enterothorax with mediastinal shift ( - Fig. 2). A left-sided chest tube was inserted, which drained a fluid that was initially considered to be old blood. Due to the sudden onset of symptoms and a normal chest X-ray which was available from the age of 1 year ( - Fig. 3 ), a diaphragmatic rupture was considered as a differential diagnosis. The boy was therefore taken to the operation room (OR) immediately. On diagnostic laparoscopy, a left-sided Bochdalek hernia was detected with herniation of the small intestine, spleen, and stomach into the chest ( - Fig. 4). Bile-stained fluid

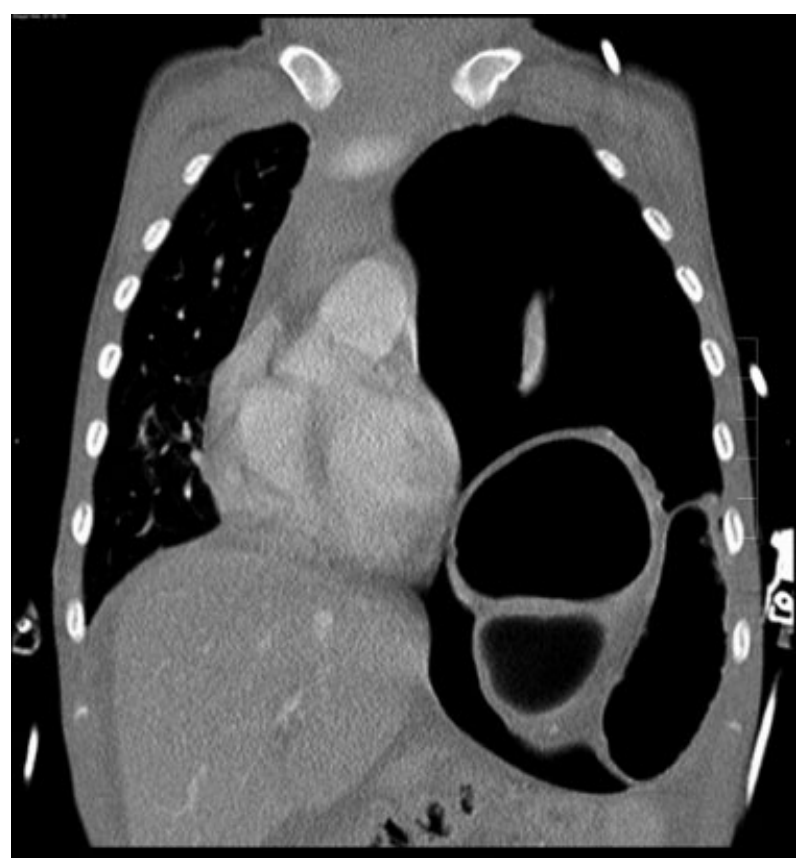

Fig. 2 Thoracic CT scan on admission. Left-sided tension pneumothorax with mediastinal shift. No further signs of a traumatic etiology. CT, computed tomography.

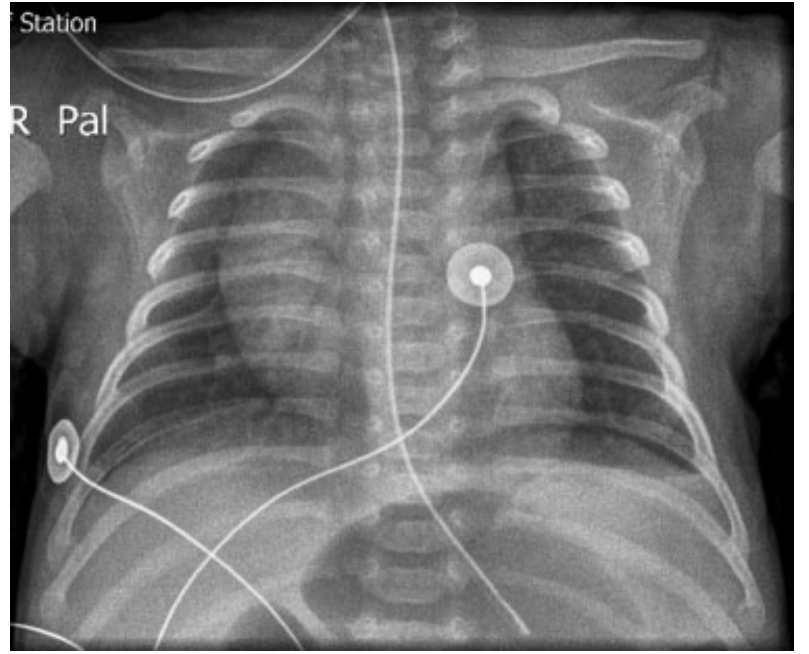

Fig. 3 Chest X-ray at 1-year of age with no signs of diaphragmatic hernia.

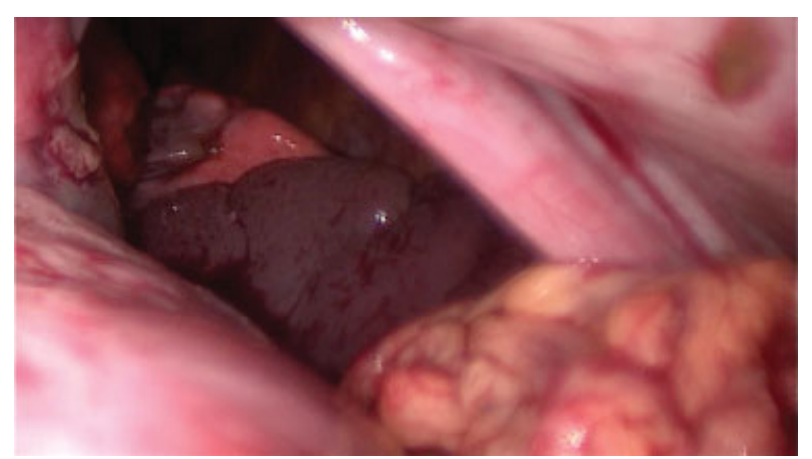

Fig. 4 Diagnostic laparoscopy: herniation of stomach, spleen, and bowel into the chest.

was found in the thorax and abdomen. After repositioning of the herniated organs into the abdomen, a gastric perforation at the lesser curvature was detected ( - Fig. 5), explaining the pneumothorax. The surgeon felt that the gastric perforation could not be closed safely laparoscopically; therefore, a conversion to laparotomy was performed with closure of the gastric perforation and repair of the $\mathrm{CDH}$ with interrupted stitches. After extubation on the fourth postoperative day, a retrovesical abscess was drained 30 days after the surgery. Due to a

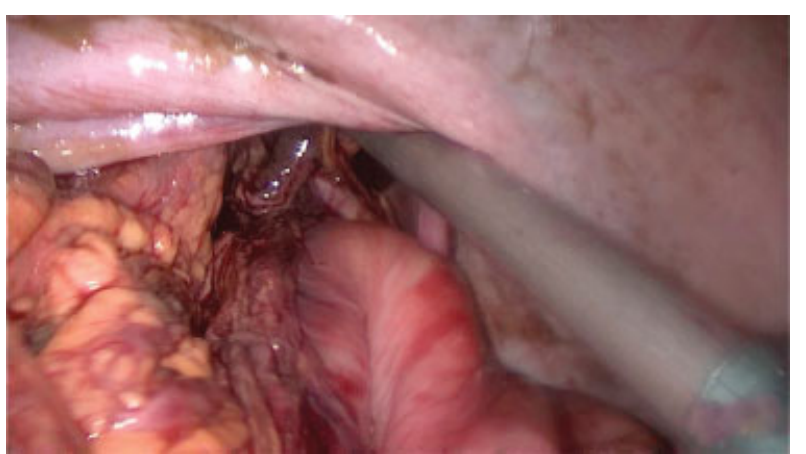

Fig. 5 Gastric perforation after repositioning of herniated organs into the abdomen. 
gastroparesis, the boy showed a prolonged recovery and was finally discharged after 4 to 5 weeks in good condition. After a follow-up of 2 years, the boy is asymptomatic and is doing well.

\section{Discussion}

Intestinal symptoms of late presenting $\mathrm{CDH}$ can be nonspecific and may include abdominal pain or respiratory symptoms. ${ }^{3,8}$ On radiologic imaging, heart murmurs and dilated bowel loops or intrathoracic cysts ${ }^{3}$ are not found infrequently. We present the second case of a $\mathrm{CDH}$ with tension pneumothorax due to gastric perforation. $\mathrm{CDH}$ in association with gastric morbidity has been described in children of different age groups. In a systemic review on 362 children with late-presenting $\mathrm{CDH}$, $46 \%$ of these were $<1$ year old, $32 \%$ aged 1 to 5 years, and $22 \%$ over 5 years old. ${ }^{6}$ Therefore, the patient presented here is in the middle spectrum of these age groups. Gastric complications of $\mathrm{CDH}$ include gastric volvulus ${ }^{7}$ and an incarceration of the stomach with or without perforation. ${ }^{4}$ Baglai et al reported on gastric volvulus being the leading cause of gastric morbidity in late-presenting $\mathrm{CDH}$ in $45 \%$ followed by gastric incarceration with (35\%) and without (20\%) perforation. ${ }^{6}$ In our case, we speculate that gastric ischemia with perforation in the absence of gastric volvulus was most likely the pathogenesis. However, we cannot rule out a combination of a previous volvulus and subsequent gastric perforation.

Unusual in our case was the clinical presentation with tension pneumothorax, which has been described before; Ozkan et $\mathrm{al}^{5}$ reported a 5-year-old girl in whom the initial chest X-ray showed a thoracic herniation of the stomach, which was misinterpreted as a lung cyst at the left lower lobe. Two days after discharge, she was readmitted for a left-sided tension pneumothorax. This complication is extremely rare in $\mathrm{CDH}$. In hindsight, as the chest tube in our patient drained the same fluid as did the nasogastric (NG) tube, we could have already thought of a hollow visceral perforation preoperatively. As the second imaging modality, a thoracic CT scan was performed as rupture of the diaphragm was considered a possible differential diagnosis. However, it can be discussed whether the CT scan was necessary, since it did not add additional information to the findings revealed by a conventional X-ray.

$\mathrm{CDH}$ may be asymptomatic at birth and may present at a later age. The herniation of the viscera into the chest leads to respiratory symptoms. In case of both, enterothorax and tension pneumothorax, a gastric or bowel perforation in combination with $\mathrm{CDH}$ must be taken into consideration.

\section{Conflict of Interest}

None.

\section{Acknowledgment}

We acknowledge support from the German Research Foundation (DFG) and Leipzig University within the program of Open Access Publishing.

\section{References}

1 Dalencourt G, Katlic MR. Abdominal compartment syndrome after late repair of bochdalek hernia. Ann Thorac Surg 2006;82 (02):721-722

2 Malekzadegan A, Sargazi A. Congenital Diaphragmatic Hernia with Delayed Presentation. Case Rep Surg [Internet]. Hindawi Publishing Corporation; 2016;2016:1-4. Available from: https://www.hindawi.com/journals/cris/2016/7284914/

3 Anaya-Ayala JE, Naik-Mathuria B, Olutoye OO. Delayed presentation of congenital diaphragmatic hernia manifesting as combined-type acute gastric volvulus: a case report and review of the literature. J Pediatr Surg 2008;43(03):E35-E39

4 Bagłaj M. Late-presenting congenital diaphragmatic hernia in children: a clinical spectrum. Pediatr Surg Int 2004;20(09):658-669

5 Ozkan A, Bozkurter Cil AT, Kaya M, Etcioglu I, Okur M. Late presenting Bochdalek hernia with gastric perforation. Pediatr Emerg Care 2015;31(01):47-49

6 Bagłaj M, Dorobisz U. Late-presenting congenital diaphragmatic hernia in children: a literature review. Pediatr Radiol 2005;35 (05):478-488

7 Ito TE, Hasnie R, Crosby DL, Milbrandt JC, Ettema S, Duong M. Gastric volvulus complication in an infant with undiagnosed congenital diaphragmatic hernia presenting with acute respiratory distress. Pediatr Emerg Care 2012;28(10):1078-1080

8 Muzzafar S, Swischuk LE, Jadhav SP. Radiographic findings in latepresenting congenital diaphragmatic hernia: helpful imaging findings. Pediatr Radiol 2012;42(03):337-342 